Rev. Bras. Saúde Prod. Anim., Salvador, v.16, n.2, p.449-457 abr./jun.., 2015 http://www.rbspa.ufba.br ISSN 15199940

\title{
Avaliação de diferentes doses de eCG na ressincronização da ovulação em vacas nelore lactantes submetidas à IATF
}

\author{
Evaluation of different eCG doses in resynchronization of ovulation in lactating nelore \\ cows subjected to fixed-time insemination
}

DOROTEU, Emanuel Mourão; OLIVEIRA, Rodrigo Arruda de"; PIVATO, Ivo ${ }^{1}$

'Universidade de Brasília, Brasília, Distrito Federal, Brasil.

*Endereço para correspondência: emanuel_doroteu@hotmail.com

\section{RESUMO}

O objetivo foi avaliar o uso da eCG na ressincronização (RS) em vacas Nelore lactantes, conhecer a interação entre dose, presença ou não de cio, presença ou não do corpo lúteo (CL) e taxa de prenhez. Foram utilizadas 775 vacas na $1^{\text {a }}$ IATF e as vazias (449) foram ressincronizadas após 30 dias. Realizou-se exame ultrassongráfico (US) antes da primeira IATF e no momento da RS para observar a presença ou não do CL. No dia 0 (D0) foi inserido dispositivo intravaginal (DIV) com $1 \mathrm{~g}$ de progesterona e aplicação intramuscular (IM) de $2 \mathrm{mg}$ de Benzoato de Estradiol. No D8 o DIV foi retirado, aplicado IM $1 \mathrm{mg}$ de cipionato de estradiol e $0,53 \mathrm{mg}$ de cloprostenol sódico. No D8 o Grupo-0 (G0) não recebeu aplicação de eCG, o G200 recebeu 200 UI e o G300 300 UI. Foi observado cio entre a retirada do DIV e a IATF. Todas as vacas foram inseminadas 48 horas após a retirada do DIV. O US foi realizado 30 dias após a IATF. Os tratamentos não alteraram a ocorrência de prenhez nas que apresentaram cio. Nas vacas que não apresentaram cio, não existe houve diferença na ocorrência de prenhez entre o G200 e o G300, mas sim com o controle. A taxa acumulada de prenhez após 40 dias da estação de monta foi de $77,54 \%$. A taxa acumulada de prenhez em 40 dias viabiliza a RS como uma forma de otimizar o manejo reprodutivo nas fazendas de corte.

Palavras-chave: eCG, estação de monta, IATF, pós-parto, ressincronização

\section{SUMMARY}

The aim was to evaluate the use of eCG in resynchronization (RS) in lactating Nelore cows, understand the interaction between dose, presence or absence of estrus, presence or absence of the corpus luteum (CL) and pregnancy rate. Were used 775 cows in first TAI and that not pregnant (449) were resynchronized 30 days after. Ultrasound (US) to observe the presence or absence of CL was performed before first TAI and when the RS. On day 0 (D0) an intravaginal device (IVD) with $1 \mathrm{~g}$ of progesterone and intramuscular (IM) $2 \mathrm{mg}$ estradiol benzoate was administered. On D8 devices were removed and applied IM 1mg estradiol cypionate; $0.53 \mathrm{mg}$ of sodium cloprostenol, Group - 0 (G0) didn't received eCG, G200 received 200 IU and G300 300 IU. Estrus was observed and insemination (AI) performed 48 hours after the removal of the IVD. US was performed 30 days after AI. The treatments didn't affect the occurrence of pregnancy in estrus. In cows that didn't show estrus, there's no difference in the occurrence of pregnancy between the G200 and G300 but with G0. The cumulative pregnancy rate after 40 days of the breeding season was $(77.54 \%)$. The cumulative pregnancy rate in 40 days enables the $\mathrm{RS}$ as a way to optimize reproductive management on beef cattle farms.

Keywords: breeding season, eCG, FTAI, postpartum, resynchronization 
Rev. Bras. Saúde Prod. Anim., Salvador, v.16, n.2, p.449-457 abr./jun.., 2015 http://www.rbspa.ufba.br

\section{INTRODUÇÃO}

O alto desempenho reprodutivo é essencial para garantir a produção máxima do rebanho e retorno econômico satisfatório. A inclusão de programas de reprodução é importante na rotina de fazendas de cria, principalmente, para aumentar a rentabilidade cujo principal objetivo é a produção de bezerros (BARUSELLI et al., 2012).

A fim de proporcionar uma segunda chance para as vacas que foram diagnosticadas como vazias em programas de IATF, o uso da ressincronização tem sido difundido nas criações extensivas de gado de corte. A utilização da IATF associada à ressincronização como manejo estratégico aumenta a proporção de animais prenhes na primeira metade da estação de monta (produção de um bezerro/vaca/ano), e consequentemente a quantidade de bezerros oriundos de IA (FREITAS et al., 2007; MARQUES et al., 2012).

Vacas com balanço energético negativo após o parto e a influência da amamentação e baixa condição corporal são fatores determinantes para o aumento do período do anestro pósparto. Nessas condições, há ausência ou inadequada pulsatilidade de LH causando efeitos negativos no estágio final do desenvolvimento folicular e na ovulação. Além disso, as fêmeas zebuínas apresentam alta incidência de cio durante a noite dificultando sua detecção (SHORT et al., 1990; BÓ et al., 2003; BARUSELLI et al., 2004). Sendo assim, um método para melhorar a taxa de serviço de vacas zebuínas seria o uso de tratamentos hormonais que estimulam o desenvolvimento folicular e a sincronização da ovulação permitindo a IATF (CAMPOS et al., 2013).

Um dos tratamentos mais utilizados para a IATF e a ressincronização consiste na inserção do dispositivo intravaginal de progesterona, aplicação via intramuscular de benzoato de estradiol, prostaglandina, eCG e cipionato de estradiol (ELZARKOUNY et al., 2004). A administração de eCG no momento da retirada do dispositivo em um protocolo com progesterona e estradiol, aumenta a taxa de ovulação e do crescimento do folículo ovulatório, em vacas de corte em anestro, e induz a formação de um corpo lúteo maior com capacidade de produzir mais progesterona. Assim o uso do eCG tem sido adotado a fim de melhorar a eficiência reprodutiva em animais no período pós parto ou com baixa condição corporal (BÓ et al., 2002; BARUSELLI et al., 2008; NÚÑEZ-OLIVEIRA et al., 2014).

Tendo em vista esses aspectos, objetivou-se avaliar diferentes doses de eCG na ressincronização em vacas da raça Nelore lactantes submetidas à IATF.

\section{MATERIAL E MÉTODOS}

O presente estudo foi aprovado pela comissão de ética no uso animal (CEUA) do Instituto de Ciências Biológicas da Universidade de Brasília com UnBDOC n ${ }^{\circ} 136533 / 2013$.

$\mathrm{O}$ experimento foi conduzido em uma fazenda situada no município da Chapada Gaúcha - MG, noroeste mineiro, entre os meses de janeiro a março de 2013. Foram utilizadas 775 vacas, devidamente identificadas, da raça Nelore (Bos indicus) pluríparas, paridas entre 35 e 75 dias mantidas com os bezerros. Todos os animais foram 
Rev. Bras. Saúde Prod. Anim., Salvador, v.16, n.2, p.449-457 abr./jun.., 2015 http://www.rbspa.ufba.br ISSN 15199940

mantidos sob pastejo (Brachiaria brizantha, cultivar Marandú) suplementadas com sal mineral e acesso livre a água. $\mathrm{O}$ escore corporal médio dessas fêmeas foi de 3,0 (escala de 1 a $5 ; 1=$ muito magra e $5=$ muito gorda). No início do protocolo da IATF (dia zero $=\mathrm{D} 0)$, as 775 vacas foram classificadas, através de exame ultrassonográfico, como cíclicas ou acíclicas (presença ou não de corpo lúteo). Utilizou-se equipamento de ultrassom Mindray (DP 2200vet, transdutor linear $7,5 \mathrm{MHz}$, China). Todos os animais do experimento receberam o mesmo protocolo da primeira sincronização que consistiu na inserção de um dispositivo intravaginal novo de $1 \mathrm{~g}$ de progesterona e $2 \mathrm{mg}$ de benzoato de estradiol por via intramuscular (IM). Oito dias depois (D8) os dispositivos foram retirados e aplicados simultaneamente $1 \mathrm{mg}$ de cipionato de estradiol; 0,530mg de cloprostenol sódico e 300 UI de eCG IM. Todas as vacas foram inseminadas 48 horas após a retirada do dispositivo intravaginal (dia $10=\mathrm{D} 10)$.

$\mathrm{O}$ diagnóstico de gestação e a segunda observação dos corpos lúteos (CL) foram realizados através da ultrassonografia transretal 30 dias após a primeira IATF. Das 775 vacas, 449 vacas $(57,9 \%)$ foram diagnosticadas como vazias e participaram da ressincronização. Foram homogeneamente alocadas em 3 grupos experimentais de forma equilibrada. Todos os animais foram inseminados pelo mesmo técnico. O sêmen utilizado no presente estudo foi analisado antes da primeira IATF e da ressincronização quanto à motilidade, vigor, turbilhonamento e características morfológicas de acordo com as normas do Colégio Brasileiro de Reprodução Animal. Todos os grupos foram inseminados com sêmen da mesma partida e do mesmo touro. Os animais do grupo controle, G-0 $(\mathrm{n}=148)$, não receberam eCG. Já aqueles dos grupos G-300 (n=150) e G-200 (n=151) receberam 300 UI e 200UI de eCG respectivamente no momento da retirada do implante. (Figura 1). A fim de se observar o cio no dia da inseminação, fez-se o uso apenas de um bastão marcador de estro na região lombo-sacra. $\mathrm{O}$ último exame ultrassonográfico foi realizado após 30 dias da ressincronização.

Empregou-se o teste da razão de verossimilhança para determinar a importância de cada variável ou interação no modelo. Considerou-se significativo $\mathrm{P}<0,05$ e o programa Statistical Analysis System (SAS, 2008) 9.2 foi empregado na análise. Para se comparar a proporção de vacas entre os com corpos lúteos, usou-se o teste de McNemar.

No resultado do teste de razão de verossimilhança, verificou-se que $o$ modelo que melhor ajusta-se aos dados é com a presença dos efeitos individuais (dose, cio e corpo lúteo) e as interações com duas variáveis (cio com dose e cio com corpo lúteo). Esse modelo indica que a associação entre dose e prenhez não é a mesma para os animais que apresentaram cio e aqueles que não apresentaram cio. Além disso, o modelo ajustado indica que a relação entre corpo lúteo e dose não apresenta significância na probabilidade de prenhez $(\mathrm{P}=0,2133)$. 
Rev. Bras. Saúde Prod. Anim., Salvador, v.16, n.2, p.449-457 abr./jun.., 2015 http://www.rbspa.ufba.br
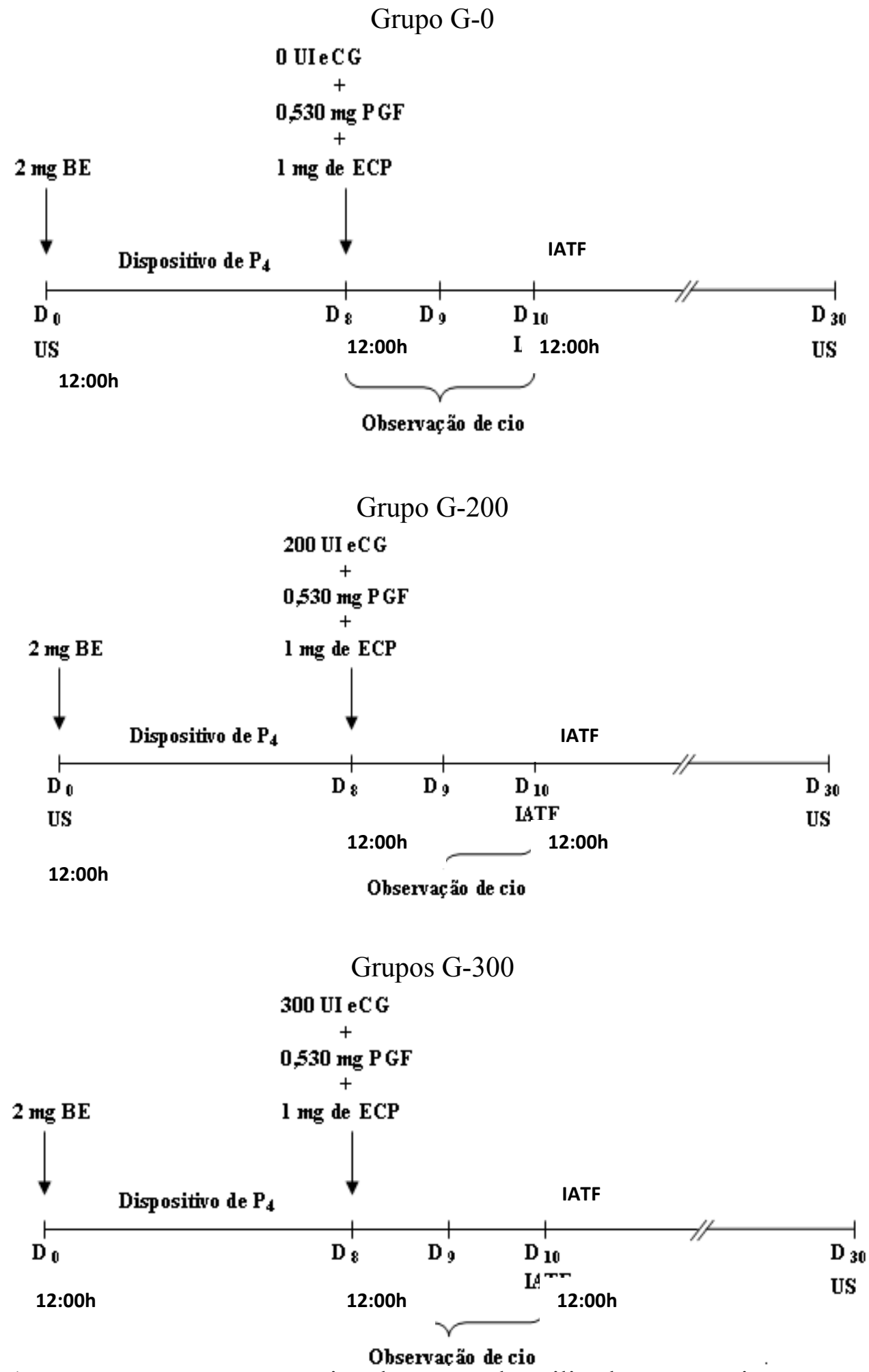

Figura 1 Representação esquemática do protocolo utilizado no experimento em vacas Nelore lactantes. Após 30 dias da primeira IATF foi realizado o diagnóstico precoce de gestação, visualização da presença ou ausência do corpo lúteo e início da ressincronização nas vacas vazias. No D8 o implante de P4 foi retirado e aplicado 0,530mg de cloprostenol (PGF), $1 \mathrm{mg}$ de ECP e as doses de 200, 300 ou 0 UI de eCG nos diferentes grupos. O cio foi observado do D8 ao D10. A IATF foi realizada no D10 
Rev. Bras. Saúde Prod. Anim., Salvador, v.16, n.2, p.449-457 abr./jun.., 2015 http://www.rbspa.ufba.br

\section{RESULTADOS E DISCUSSÃO}

Nos animais que apresentaram cio após a retirada do dispositvo de $\mathrm{P} 4$, os diferentes tratamentos (0 UI, 200 UI e 300 UI de eCG) não alteraram significativamente a ocorrência de prenhez. Além disso as diferentes doses não diferem significativamente entre si. Nos animais que não apresentaram cio a dose de 200 UI de eCG apresentou 3,035 vezes mais chances de melhorar a taxa de prenhez do que o grupo controle. Animais com dose de 300 UI de eCG apresentaram 2,893 vezes mais chances de prenhez em relação ao grupo controle (Tabela 1). Não existe diferença significativa na ocorrência de prenhez nos animais que receberam a dose de 200 UI ou 300 UI. Nos gráficos 1 e 2 é possível observar o comportamento diferente entre as vacas que apresentaram cio daquelas que não apresentaram.

Tabela 1. Efeito da dose e presença ou não de cio na taxa de prenhez em vacas Nelore ressincronizadas submetidas à IATF. Chapada Gaúcha - MG, janeiro a março/2013

\begin{tabular}{llcccc}
\hline Dose & Cio & Razão de chance & Intervalo de $95 \%$ de confiança & p-valor \\
\hline 200 vs 0 & não & 3.035 & 1.201 & 7.66 & 0,0189 \\
300 vs 0 & não & 2.893 & 1.018 & 8.221 & 0,0461 \\
200 vs 300 & não & 1.049 & 0.393 & 2.799 & 0,9243 \\
200 vs 0 & sim & 0.557 & 0.302 & 1.026 & 0,0603 \\
300 vs 0 & sim & 0.912 & 0.490 & 1.699 & 0,7720 \\
200 vs 300 & sim & 0.611 & 1.201 & 1.079 & 0,0893 \\
\hline P- valor $<0,05$. & & & &
\end{tabular}

O uso de 200 UI do eCG (Folligon ${ }^{\circledR}$, Intervet/Schering-Plough, Brasil) gera uma diferença de aproximadamente $\mathrm{R} \$$ 3,20 no protocolo de inseminação em relação a dose de 300 UI. Assim, além das vantagens genéticas e de logística quanto ao nascimento dos bezerros, a ressincronização pode ser realizada por um menor custo sem causar impacto na probabilidade de prenhez quando a dose de eCG for reduzida (Figuras 2 e 3 ).

As vacas que ficaram vazias após a primeira IATF com presença de corpo lúteo, verificado no início da sincronização $\quad(16,26 \%)$ é significativamente menor daquelas com ausência de corpo lúteo verificado no início da ressincronização $(67,26 \%)$ como pode ser observado na Tabela 2 . Resultado semelhante (16,8\%) encontrado por Vasconcelos et al. (2006) quando foram utilizados animais sob as mesmas condições do presente estudo. Após 30 dias, no momento do início da ressincronização, foi observado que $67,26 \%(\mathrm{P}<0,05 \%)$ das fêmeas saíram do anestro a voltaram à ciclicidade, possivelmente devido a indução da ciclicidade após a primeira IATF como verificado por Bó et al. (2002). 
Rev. Bras. Saúde Prod. Anim., Salvador, v.16, n.2, p.449-457 abr./jun.., 2015 http://www.rbspa.ufba.br

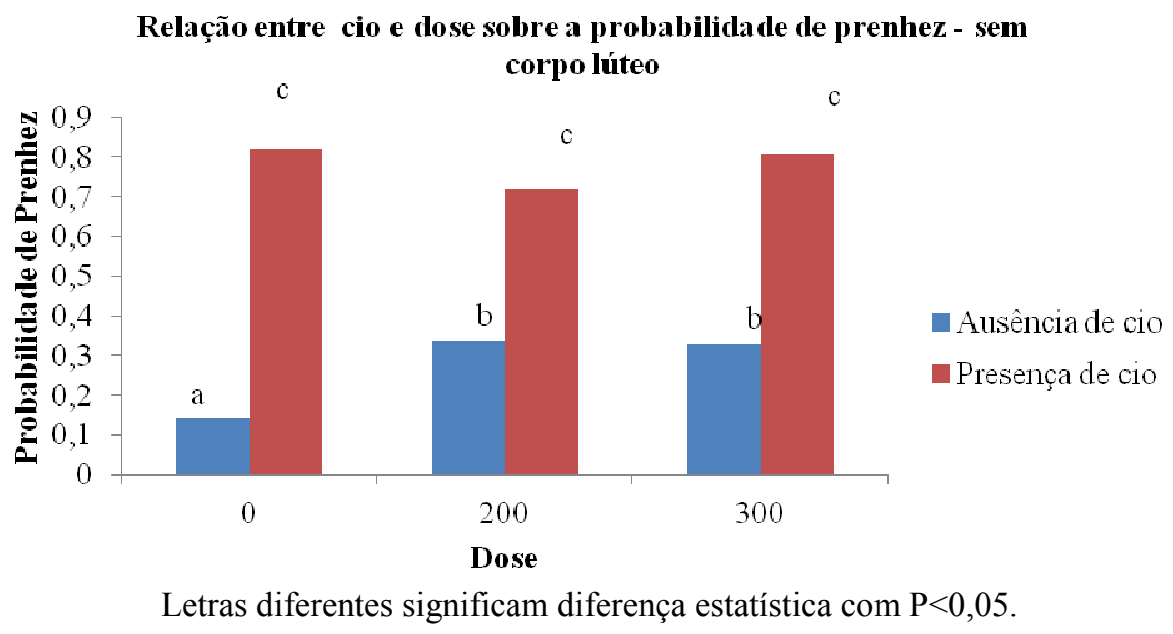

Figura2. Influência da presença de cio e da dose de eCG na probabilidade de prenhez em vacas Nelores lactantes, com ausência de corpo lúteo, em programa de resincronização para IATF.Chapada Gaúcha - MG, janeiro a março/2013

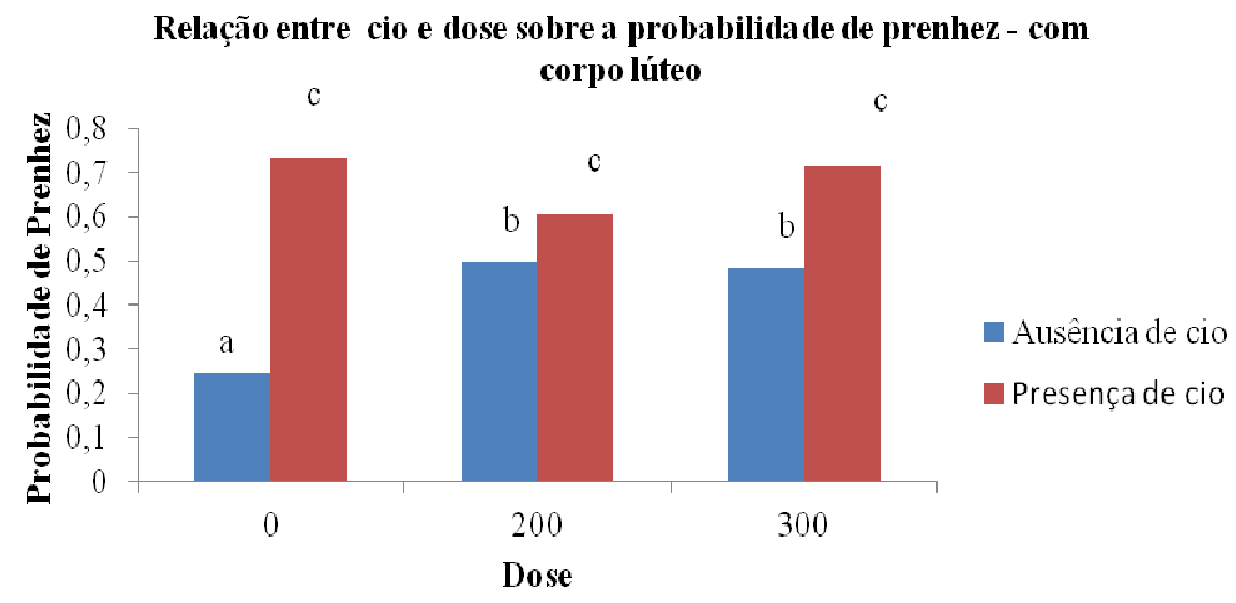

Letras diferentes significam diferença estatística com $\mathrm{P}<0,05$.

Figura 3.Influência da presença de cio e da dose de eCG na probabilidade de prenhez em vacas Nelore lactantes, com presença de corpo lúteo, em programa de resincronização para IATF.Chapada Gaúcha - MG, janeiro a março/2013

Tabela 2. Presença ou não de corpo lúteo no momento da seleção de vacas Nelore submetidas aos protocolos de sincronização e ressincronização para IATF.Chapada Gaúcha - MG, janeiro a março/2013

\begin{tabular}{cccccc}
\hline \multicolumn{3}{c}{ Sincronização } & \multicolumn{3}{c}{ Ressincronização } \\
\hline Com CL n (\%) & Sem CL n (\%) & Total n (\%) & Com CL n (\%) & Sem CL n (\%) & Total n (\%) \\
$73(16,26)^{\mathrm{a}}$ & $376(83,74)^{\mathrm{b}}$ & $449(100)$ & $302(67,26)^{\mathrm{c}}$ & $147(32,74)^{\mathrm{d}}$ & $449(100)$ \\
\hline
\end{tabular}

Letras diferentes na mesma linha indicam diferença estatística com $\mathrm{P}<0,05$. 
Rev. Bras. Saúde Prod. Anim., Salvador, v.16, n.2, p.449-457 abr./jun.., 2015 http://www.rbspa.ufba.br ISSN 15199940

Além dos fatores ambientais e individuais, a amamentação e a condição nutricional são importantes para o desencadeamento do anestro pósparto. A maior necessidade energética na vida de vacas adultas acontece após o parto, pois o dispêndio de energia é voltado para a manutenção dos sinais vitais e produção de leite (OILVEIRA et al., 2010). Uma estratégia para diminuir o intervalo entre partos em vacas de corte no pós-parto é a utilização da eCG após a retirada do implante de progesterona. Alguns estudos demonstram que a eCG provoca aumento no crescimento folicular, aumento na taxa de ovulação e de concepção (KASTELIC et al., 1999; BARUSELLI et al., 2008; BARREIROS et al., 2014; NÚÑEZOLIVEIRA et al., 2014).

O desmame temporário também é uma estratégia para melhorar a eficiência reprodutiva em vacas Nelore após o parto devido ao aumento na frequência dos pulsos de $\mathrm{LH}$, pois a remoção do contato visual e olfatório diminui os efeitos negativos da amamentação na secreção de LH (STAGG et al., 1998; BARREIROS et al., 2014). Em um protocolo de ressincronização de 23 dias, Campos et al. (2013) além de avaliar a eficiência de 0,200 ou 300 UI de eCG, realizaram desmame temporário de 48 a $54 \mathrm{~h}$ em vacas Nelore paridas entre 64 a 90 dias e observaram que os resultados da dose de 300 UI e o desmame temporário foram semelhantes na taxa de prenhez, aumentando a eficiência reprodutiva desses animais.

Como observado na Tabela 3 , ao final da estação de monta, em 40 dias foi possível obter uma taxa de prenhez na ressincronização de $61,24 \%(275 / 449) \mathrm{e}$ acumulada de 77,54\% (601/775). Assim, possibilita alcançar grande número de vacas prenhes no início da estação, favorecendo a organização da propriedade na época dos nascimentos que serão de forma concentrada e de possibilitar mais chance para aquelas fêmeas que não ficaram gestantes. $O$ impacto da concepção no início da estação monta está relacionado à rentabilidade na comercialização por peso dos bezerros, pois serão mais velhos e pesados no momento da desmama (LESMEISTER et al., 1973). Resultado semelhante encontrado por Marques et al. (2012) ao trabalharem com vacas Nelore pluríparas paridas na ressincronização 30 dias após a primeira IATF em estação de monta de 40 dias alcançando 79,3\% de prenhez.

Tabela 3. Taxa de prenhez obtida na IATF, na ressincronização e a taxa acumulada total ao final da estação de monta em 40 dias.Chapada Gaúcha - MG, janeiro a março/2013

\begin{tabular}{lcc}
\hline Técnica & $\mathrm{N}^{\circ}$ animais & Prenhez $\mathrm{n}(\%)$ \\
\hline IATF & 775 & $326(42,06)$ \\
Ressincronização & 449 & $275(61,24)$ \\
Taxa acumulada (\%) & - & $601(77,54)$ \\
\hline
\end{tabular}

As técnicas para melhorar a eficiência reprodutiva e o ganho genético dos bovinos são fatores determinantes para aumentar a produtividade e o retorno econômico da pecuária de corte. A utilização da IATF com a ressincronização possibilita obter maior número de bezerros oriundos de 
Rev. Bras. Saúde Prod. Anim., Salvador, v.16, n.2, p.449-457 abr./jun.., 2015 http://www.rbspa.ufba.br ISSN 15199940

inseminação aos 40 dias da estação de monta e reduz o número de touros para repasse, favorece o cruzamento industrial, reposição de matrizes e maior padronização dos lotes de bezerros. A utilização de 200 UI eCG em vacas Nelore lactantes pode ser realizada na ressincronização sem causar prejuízo à probabilidade de prenhez e reduz $\mathrm{o}$ custo do protocolo.

\section{REFERÊNCIAS}

BARREIROS, T.R.; BLASCHI, W.; SANTOS, G.M.; MOROTTI, F.; ANDRADE, E.R.; BARUSELLI, OS.; SENEDA, M.M. Dynamics of follicular growth and progesterone concentrations in cyclic and anestrous sucking Nelore cows (Bos indicus) treated with progesterone, equine chorionic gonadotropin, or temporary calf removal. Theriogenology, v.81, p.651656, 2014.

BARUSELLI, P.S.; REIS, E.L.; MARQUES, M. O.; NASSER, L.F.; BÓ, G.A. The use of hormonal treatments to improve reproductive performance of anestrous beef cattle in tropical climates. Animal

Reproduction Science, v.82/83, p.479486, 2004.

BARUSELLI, P.S.; JACOMINI, J.O.; SALES, J.N.; CREPALDI, G.A.; Importância do emprego da eCG em protocolos de sincronização para IA, TE e SOV em tempo fixo. IN: SIMPÓSIO

INTERNACIONAL DE

REPRODUÇÃO ANIMAL APLICADA, 3., 2008, Londrina. Anais... Londrina, 2008. p.146-167.

BARUSELLI, P.S.; SALES, J.N.S.; SALA, R.V.; VIEIRA, L.M.; SÁ FILHO, M.F. History, evolution and perspectives of timed artificial insemination programs in Brazil. Animal Reproduction, v.9, n.3, p.139152, 2012.

BÓ, G.A.; CUTAIA, L.; TRIBUTO, R. Inseminación artificial a tiempo fijo em bovinos de carne. In: CURSO DE ABORDAGEM TEÓRICA-PRÁTICA DE NOVAS TÉCNICAS DE SINCRONIZAÇÃO SEM OBSERVAÇÃO DE CIO EM BOVINOS, 2., 2002, Cornélio Procópio, PR. Anais... Cornelio Procópio, PR, 2002. p.23-39.

BÓ, G.A.; BARUSELLI, P.S.; MARTÍNEZ, M.F. Pattern and manipulation of follicular development in Bos indicus cattle. Animal of Reproduction Science, v.78, p.307326, 2003.

CAMPOS, J.T.; MARINHO L.S.R.; LUNARDELLI, P.A.; MOROTTI, F.; SENEDA, M.M. Resynchronization of estrous cycle with eCG and temporary calf removal in lactating Bosindicus cows. Theriogenology, v.80, p.619623, 2013.

EL-ZARKOUNY, S.Z.; STEVENSON, J.S. resynchronizing estrus with progesterone or progesterone plus estrogen in cows of unknown pregnancy status. Journal of Dairy Science, v.87, p.3306-3321, 2004.

FREITAS, D.S.; CHALHOUB, M.; ALMEIDA, A.K.C.; SILVA, A.A.B.; SANTANA, R.C.M.; RIBEIRO FILHO, A.L. Associação do diagnóstico precoce de prenhez a um protocolo de ressincronização do estro em vacas zebuínas. Revista Brasileira de Saúde e Produção Animal [online], v.8, n.3, p.170-177, 2007. 
Rev. Bras. Saúde Prod. Anim., Salvador, v.16, n.2, p.449-457 abr./jun.., 2015 http://www.rbspa.ufba.br ISSN 15199940

KASTELIC, J.P. Synchronization of estrus in beef cattle with norgestomet and estradiol valerate. Canadian

Veterinary Journal, v.40, p.173-178, 1999.

LESMEISTER, J.L.; BURJENING, J.; BLACKWELL, R.L. Date of first calving in beef cows and subsequent calf production. Journal of Animal Science, v.36, p.1-6, 1973.

MARQUES, M.O.; RIBEIRO JUNIOR, M.; SILVA, R.C.P.; SÁ FILHO, M.F.; VIEIRA, L.M.; BARUSELLI, P.S. Ressincronização em bovinos de corte. In: SIMPÓSIO INTERNACIONAL DE REPRODUÇÃO ANIMAL

APLICADA, 5., 2012, Londrina.

Anais... Londrina, 2012. p.82-92.

NÚÑEZ-OLIVEIRA, R.; DE CASTRO, T.; GARCÍA-PINTOS, C.; BÓ, G.; PIAGGIO, J.; MENCHACA, A. Ovulatory response and luteal funcion after eCG administration at the endo $\mathrm{f}$ a progesterone and estradiol based treatment in postpartum anestrous beef cattle. Animal Reproduction Science. Disponível em:

$<$ http://dx.doi.org/10.1016/j.anireprosci. 2014.02.017>. Acesso em: 20 mar. 2014.

OLIVEIRA, J.F.O.; GONÇALVEZ, P.B.D.; FERREIRA, R.; GASPERIN, B.; SIQUEIRA. L.C. Controle sobre GnRH durante o anestro pós-parto em bovinos. Ciência Rural, v.40, n.12, p.2623-2631, 2010.

SHORT, R.E; BELLOWS, R.B.; STAIMGMILLER, R.B.; BERARDINELLI, J.G.; CUSTER, E.E. Physiological mechanisms controlling anestrus and infertility in postpartum beef cattle. Journal of Animal Science, v.68, p.799-816, 1990.
STAGG, K.; SPICER, L.J.; SREENAN, J.M.; ROCHE, J.F.; DISKIN, M.G.

Effect of calf isolation on follicular wave dynamics, gonadotropin and metabolic hormone changes, and interval to first ovulation in beef cows fed either of two energy levels postpartum. Biology of Reproduction, v.59, p.777-783, 1998.

STATISTICAL ANALYSIS SYSTEM

- SAS. User's guide. Version 9.2.

Cary: SAS Institute, 2008.

VASCONCELOS, J.L.M. Avaliação do protocolo de sincronização de ovulação ovsynch e de fatores relacionados à associação entre produção de leite e taxa de concepção. 1998, 128p. Tese (Doutorado) - Universidade Estadual Paulista, Faculdade de Ciências Agrárias e Veterinárias, Jaboticabal.

Data de recebimento: 28/04/2014

Data de aprovação: 26/03/2015 